\title{
Expanded Gene Panel Use for Women With Breast Cancer: Identification and Intervention Beyond Breast Cancer Risk
}

\author{
Erin O'Leary, MS, Daniela Iacoboni, MS, Jennifer Holle, MS, Scott T. Michalski, MS, Edward D. Esplin, MD, PhD, \\ Shan Yang, PhD, and Karen Ouyang, PhD
}

San Francisco, CA

\begin{abstract}
Background. Clinicians ordering multi-gene next-generation sequencing panels for hereditary breast cancer risk have a variety of test panel options. Many panels include lesser known breast cancer genes or genes associated with other cancers. The authors hypothesized that using broader gene panels increases the identification of clinically significant findings, some relevant and others incidental to the testing indication. They examined clinician ordering patterns and compared the yield of pathogenic or likely pathogenic (P/LP) variants in non-BRCA genes of female breast cancer patients.
\end{abstract}

Methods. This study analyzed de-identified personal and family histories in 1085 breast cancer cases with P/LP multi-gene panel findings in non-BRCA cancer genes and sorted them into three groups by the panel used for testing: group A (breast cancer genes only), group B (commonly assessed cancers: breast, gynecologic, and gastrointestinal), and group $\mathrm{C}$ (a more expanded set of tumors). The frequency of $\mathrm{P} / \mathrm{LP}$ variants in genes with established management guidelines was compared and evaluated for consistency with personal and family histories.

Results. This study identified $1131 \mathrm{P} / \mathrm{LP}$ variants and compared variants in clinically actionable genes for breast and non-breast cancers. Overall, $91.5 \%$ of these variants

Electronic supplementary material The online version of this article (doi:10.1245/s10434-017-5963-7) contains supplementary material, which is available to authorized users.

(C) The Author(s) 2017. This article is an open access publication

First Received: 17 April 2017;

Published Online: 1 August 2017

E. O'Leary, MS

e-mail: erin.oleary@invitae.com were in genes with management guidelines. Nearly $12 \%$ were unrelated to personal or family history.

Conclusion. Broader panels were used for $85.6 \%$ of our cohort (groups B and $\mathrm{C}$ ). Although pathogenic variants in non- $B R C A$ genes are reportedly rare, the study found that most were in clinically actionable genes. Expanded panel testing improved the identification of hereditary cancer risk. Small, breast-limited panels may miss clinically relevant findings in genes associated with other heritable cancers.

Breast cancer is a multifactorial disease caused by a combination of environmental and genetic factors. ${ }^{1}$ In an estimated $10 \%$ of breast cancers, the genetic factor is contributed primarily by mutations in a single gene. ${ }^{2}$ Testing for BRCA1 and BRCA2 (BRCA1/2) has been available for more than two decades, and multiple professional organizations have developed guidelines for testing individuals based on suggestive personal or family history. ${ }^{3-7}$

The clinical impact and cancer risks for individuals with pathogenic variants in BRCAl/2 are well studied. ${ }^{8}$ Likewise, medical management guidelines have been established and are frequently updated. ${ }^{3-5}$ These guidelines also address high- and moderate-risk genes such as TP53 and PTEN. ${ }^{3,9,10}$ Although medical management guidelines exist for many other genes such as PALB2, CHEK2, and $A T M$, no defined testing criteria have been established.

The advent of next-generation sequencing (NGS) has allowed for the efficient, cost-effective analysis of many genes simultaneously. ${ }^{11-13}$ Multiple studies have demonstrated that compared with multi-gene NGS panels, traditional testing of $B R C A 1 / 2$ alone misses potentially actionable findings in a substantial proportion of cases. ${ }^{14-18}$ The National Comprehensive Cancer Network (NCCN) and the American Society of Breast Surgeons (ASBS) address the benefits and limitations of multi-gene panels, 
but they do not explicitly advocate for or against the use this testing method. ${ }^{3,5}$ Identified benefits include the increased yield of positive findings, whereas limitations may include higher rates for variants of unknown significance and findings with no established management guidelines. ${ }^{3,5}$ In the absence of guidelines for choosing appropriately from the increasing variety of panels, oncology providers must determine whether to select highrisk panels limited to genes with established medical management guidelines or larger panels that have dozens of genes associated with a variety of non-breast hereditary cancers.

Using a consecutive series of 20,592 women with breast cancer undergoing hereditary genetic testing in our commercial laboratory, we sought to clarify the current use of larger hereditary cancer panels and determine their clinical impact. We analyzed a large cohort of breast cancer patients identified as having one or more pathogenic or likely pathogenic (P/LP) variants in genes other than $B R C A 1 / 2$. Clinician ordering patterns were reviewed, and their impact on the rate of P/LP findings was described. Personal and family history information was reviewed to determine how often genetic test results were consistent with the indications for testing and to define the utility of hereditary gene panels for identifying clinically actionable findings. We hypothesized that using broader gene panels increases the identification of clinically significant findings, some relevant and others incidental to the testing indication.

\section{METHODS}

The study cohort consisted of all patients referred to our commercial laboratory between February 2015 and August 2016 for diagnostic multi-gene panel testing for hereditary breast and ovarian cancer (HBOC). Patients referred to undergo family variant testing for a known pathogenic mutation were excluded.

The gene panels analyzed for each patient were chosen at the discretion of the ordering provider and ranged from 2 to 79 genes. The price of a panel was the same regardless of the number of genes, making test selection dependent only on patient and provider preferences. Testing was performed with NGS, as previously described, ${ }^{18}$ and variant interpretation was carried out based on an expansion of the American College of Medical Genetics and Genomics (ACMG) guidelines. ${ }^{19}$

A total of 2105 individuals with P/LP variants were identified. Of these individuals, 1020 had P/LP variants in $B R C A 1$ or $B R C A 2$ and were excluded, leaving 1085 individuals with $\mathrm{P} / \mathrm{LP}$ findings in other genes associated with increased risk of heritable cancer (Fig. 1). Notably, common and low-penetrance variants (e.g., APC I1370 K, CHEK2 I157T) were included for analysis.

According to an institutional review board (IRB)-approved study protocol, de-identified personal and family history information from submitted requisition forms and medical records (when available) for each case were reviewed and compared with published testing guidelines ${ }^{3}$ to determine whether the findings were consistent with the reported history information (expected findings) or not (incidental findings). All variants were analyzed to determine the frequency of findings in genes with published medical management guidelines, ${ }^{4,5}$ including current ASBS guidelines, NCCN guidelines for HBOC and hereditary colorectal cancer, and other published professional guidelines (see Appendix 1 in Electronic Supplementary Material).

The case series was divided into three primary order groups according to the type of panel ordered:

- Group A: panels consisting of genes associated primarily with breast cancer (ATM, BARDI, BRCAl, BRCA2, BRIP1, CDH1, CHEK2, FANCC, MRE11A, NBN, NF1, PALB2, PTEN, STK11, TP53), most of which have established clinical management guidelines. Orders in this category included up to 15 genes.

- Group B: panels containing all the genes in group A plus genes associated with other commonly assessed cancer types (i.e., breast, gynecologic, and gastrointestinal) (APC, AXIN2, BMPR1A, CDKN2A, DICER1, EPCAM, GREM1, KIT, MEN1, MLH1, MSH2, MSH6, MUTYH, PDGFRA, PMS2, POLD1, POLE, RAD51C, RAD51D, SDHA, SDHB, SDHC, SDHD, SMAD4, SMARCA4, TSC1, TSC2, VHL). Orders in this category included up to 42 genes.

- Group C: large, comprehensive panels including all the genes in groups A and B plus an expanded list of genes for other tumor types (i.e., prostate, sarcoma, brain). Orders in this category included up to 79 genes.

\section{RESULTS}

Among the 1085 cases reviewed, $1131 \mathrm{P} / \mathrm{LP}$ variants were identified, with 44 patients $(4 \%)$ having two or more $\mathrm{P} / \mathrm{LP}$ variants. Overall, $91.5 \%$ of the variants identified in this cohort were in genes with medical management guidelines (Fig. 2).

As shown in Fig. 3, the majority of cases $(72.6 \%)$ were in ordering group B. As expected, a greater proportion of $\mathrm{P} / \mathrm{LP}$ variants in genes with breast management guidelines was in group A $(97.5 \%)$ than in groups B $(63.6 \%)$ and C (50\%). In groups B and C, a significant percentage of P/LP variants (28.5 and $31.8 \%$, respectively) were identified in 


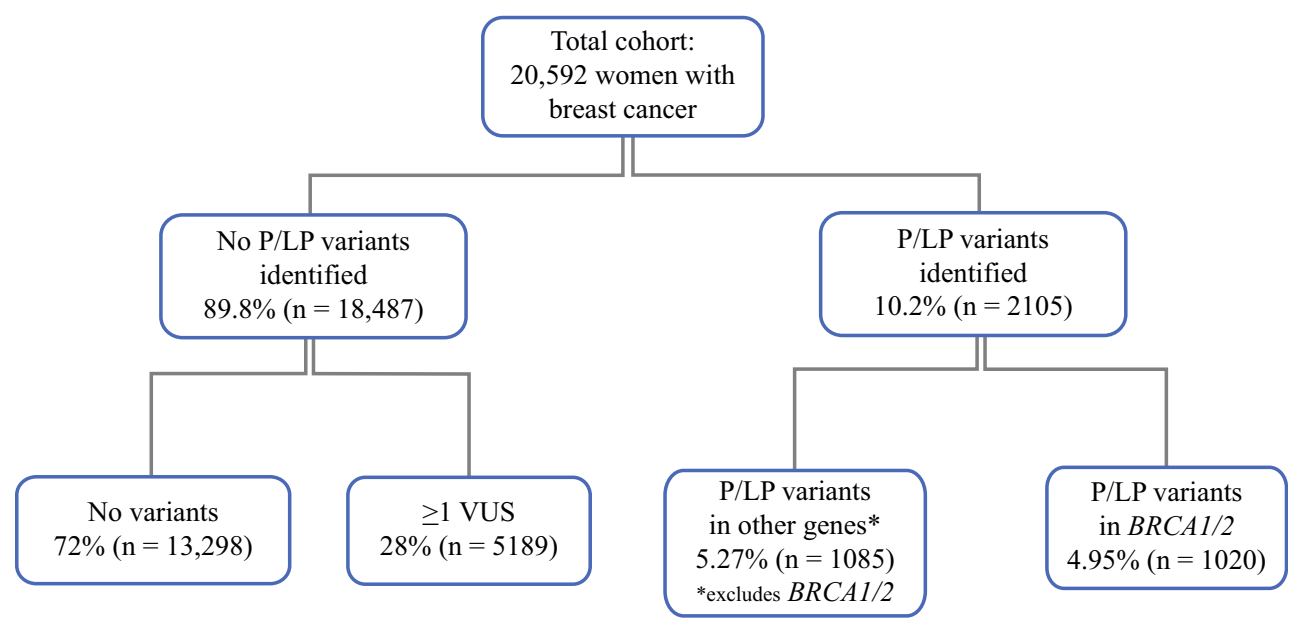

FIG. 1 Stratification of patients by variant type. Variants were identified in the patient cohort and categorized according to variant classification and BRCA1/2 status. $n$ number of patients, $P / L P$ pathogenic/likely pathogenic, $V U S$ variant of uncertain significance

1085 Patients (1131 variants)

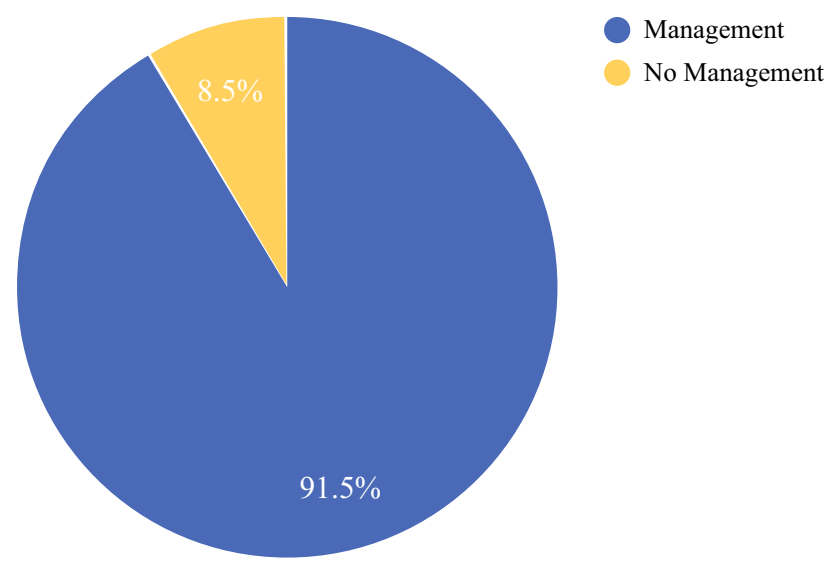

FIG. 2 Percentage of identified pathogenic and likely pathogenic variants in the total cohort of genes with medical management guidelines

genes associated with increased risk of non-breast cancers for which established management guidelines exist (see Appendix 1 in Electronic Supplementary Material).

Perhaps surprisingly, $132 \mathrm{P} / \mathrm{LP}$ variants $(11.7 \%)$ were unexpected clinically actionable findings (i.e., variants in genes for which patients had no suggestive personal or familial history and for which medical management guidelines exist). By panel, approximately 13 and $15 \%$ of the $\mathrm{P} / \mathrm{LP}$ variants identified in groups $\mathrm{B}$ and $\mathrm{C}$, respectively, consisted of these unexpected findings (Table 1).

Overall, the most common P/LP findings in BRCA1/2negative patients were in CHEK2 (27.6\%), MUTYH (15\%), ATM (14.9\%), and PALB2 (12.2\%) (Fig. 4).

We also determined the number of patients with one or more variants of uncertain significance (VUS), excluding those with both VUS and P/LP variants. As expected, VUS

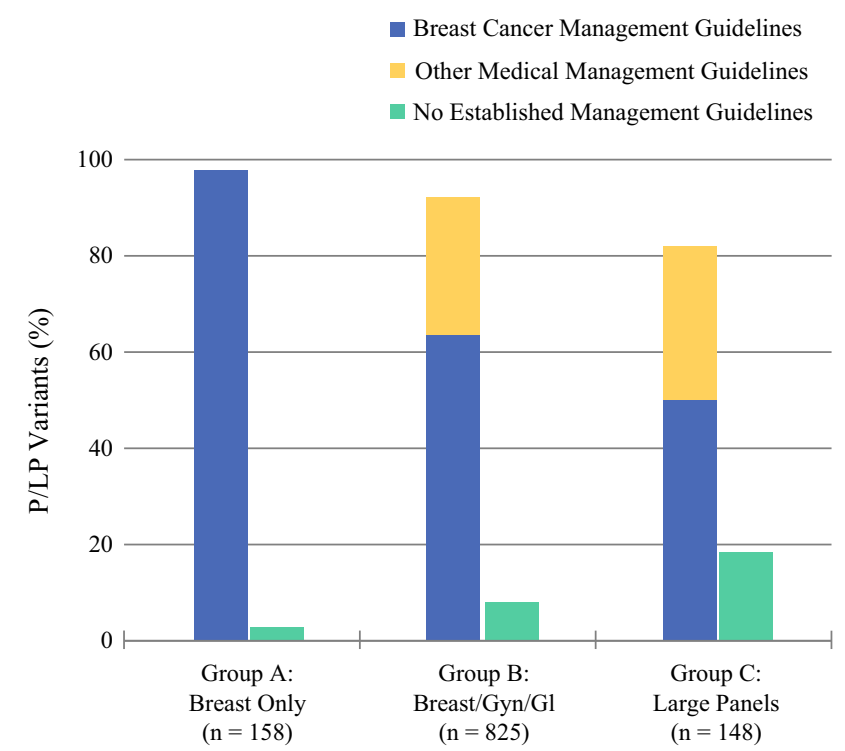

FIG. 3 Comparison of P/LP variants and associated management guidelines. $P / L P$ pathogenic/likely pathogenic, $G I$ gastrointestinal, Gyn gynecologic

rates were directly proportional to the number of genes analyzed. One VUS or more were found in $12.7 \%$ of the patients in group A, $31.6 \%$ of the patients in group B, and $49.6 \%$ of the patients in group C.

\section{DISCUSSION}

Clinicians face a multitude of options when ordering hereditary genetic testing for breast cancer risk, including single-gene tests such as those limited to $B R C A 1 / 2$, multigene panels including genes associated with a high risk of breast cancer, panels for genes associated with multiple common cancer types (e.g., breast, gynecologic, and gastrointestinal), and comprehensive panels including genes 
TABLE 1 Unexpected findings with management guidelines by panel group

\begin{tabular}{|c|c|c|c|}
\hline & Group A & Group B & Group C \\
\hline \multirow[t]{2}{*}{ Total variants } & 158 & 825 & 148 \\
\hline & & $n(\%)$ & $n(\%)$ \\
\hline Clinically actionable findings unrelated to personal or family history & Non-breast cancer genes not assessed & $110(13.3)$ & $22(14.9)$ \\
\hline
\end{tabular}

FIG. 4 Most frequently identified pathogenic and likely pathogenic variants

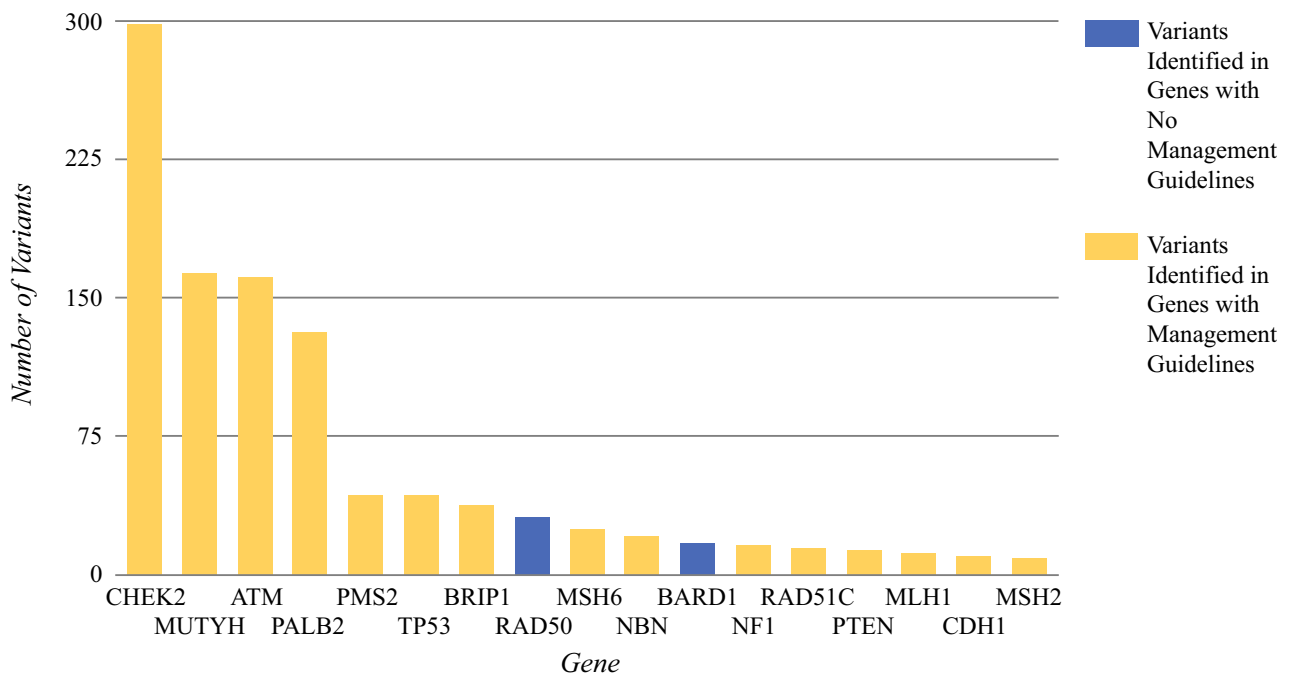

associated with both common (e.g., prostate) and rare (e.g., sarcoma) cancers. Professional societies have published testing recommendations limited to specific genes such as $B R C A 1 / 2$ and those associated with well-described hereditary cancer conditions such as Li-Fraumeni (TP53) and Cowden syndrome $(P T E N){ }^{3,5}$

The results of this report and others, however, suggest a trend toward ordering larger panels, ${ }^{20-22}$ which have the advantage of increased yield afforded by querying more genes. A recent study examining the yield of 348 multigene tests not limited to breast cancer found that the inclusion of moderate-risk genes increased only the positive rate of "historically high-risk" cancer genes. ${ }^{26}$ Another study, which assessed 90 HBOC patients, found that the positive rate roughly doubled when the panel included genes beyond $B R C A 1 / 2 .^{27}$ Among clinic-based studies of $B R C A 1 / 2$-negative patients, the prevalence of pathogenic variants in non-BRCA genes ranged from 3.7 to $16 \% .^{14-17,28-31}$

Our data support previous studies showing that expanded panel testing improves the identification of hereditary cancer risk for patients and their family members. We found that the use of expanded panels increased the number of clinically relevant results, many of which were not associated with personal or family history. In group $\mathrm{A}$, the genes tested were limited to those associated with breast cancer, and therefore, all the variants identified were related to clinical or family history. In group B, however, patients were tested for genes associated with common cancer types (including breast, gynecologic, and gastrointestinal), and $13 \%$ of the P/LP variants were identified in genes that had published medical management recommendations but were not associated with the personal or family history. This number increased to $15 \%$ in group $\mathrm{C}$, which represented the most comprehensive panels (Table 1). This proportion of unexpected findings is higher than previous estimates (8\% in a cohort of 1046 BRCA1/2negative individuals evaluated for $\mathrm{HBOC}) .^{32}$

Our findings are consistent with prior studies suggesting that expanded panels increase diagnostic yield and furthermore showed that the vast majority of variants identified $(92 \%)$ were associated with medical management guidelines that may alter clinical care. Additionally, our results suggest that breast cancer-specific panels may miss clinically actionable findings in genes associated with hereditary predispositions for other cancers.

Another important finding was that a considerable number of patients had more than one pathogenic variant. Of 44 patients with two or more clinically significant variants, $26(59 \%)$ would not have been identified with a panel specific to breast cancer.

The findings showed P/LP variants in a variety of genes (Fig. 4), but most commonly in $C H E K 2$, which accounted for $27.6 \%$ of our positive findings. This gene is associated 
with an increased risk for autosomal dominant adult-onset cancers including breast, colon, thyroid, and prostate cancers, and possibly others. ${ }^{33-37}$ These cancer risks, particularly the risks for breast cancer, depend on the variant and family history. ${ }^{34,38}$ Several studies have found that pathogenic variants in $C H E K 2$ are observed at a frequency higher than those of variants identified in other breast cancer-associated genes on multi-gene panels, ${ }^{22,26,39}$ which is consistent with our observations. One case example from our cohort was a woman who received a diagnosis of breast cancer at age 37 . Her family history was significant for breast cancer in her mother (diagnosed when she was 47 years old) and maternal aunt (diagnosed when she was 49 years old). Although the patient had previous negative $B R C A 1 / 2$ testing, her clinician ordered a larger breast cancer panel based on her age at diagnosis and striking family history. The results showed a pathogenic variant in $C H E K 2$.

Professional management guidelines include consideration of bilateral mastectomy or screening with breast magnetic resonance imaging (MRI) and colonoscopy, beginning at the age of 40 years. ${ }^{3,5}$ The use of BRCA1/2 testing alone failed to provide an explanation for the breast cancers in this family. Expanded multi-gene testing showed clinically actionable results that not only explained the family history but also had implications for family members.

In this study, MUTYH heterozygotes accounted for $15 \%$ of our P/LP findings, likely due to the relatively high MUTYH carrier frequency among whites of Northern European descent. Approximately $1.5-2 \%$ of the individuals in this population carry one of two well-described founder variants: c.536A $>\mathrm{G}$ (p.Tyr179Cys) in exon 7 and c. $1187 \mathrm{G}>\mathrm{A}$ (p.Gly396Asp). ${ }^{40-42}$ It is known that MUTYH heterozygotes have an increased risk for colorectal cancer, and medical management and surveillance protocols have been developed. ${ }^{4}$ Colonoscopy every 5 years is recommended beginning at age 40 or 10 years earlier than the youngest age at diagnosis in an affected first-degree relative. An example from our cohort was a 40-year-old woman with a diagnosis of invasive ductal carcinoma and no family history of cancer. A 26-gene breast cancer panel identified a single pathogenic MUTYH variant. Although this result may not explain her early onset breast cancer diagnosis, it showed an increased risk for colon cancer, thereby allowing the implementation of screening guidelines.

The criticisms of multi-gene panels are well known. One criticism focuses on the increased risk of obtaining uncertain results lacking clear management guidelines, such as variants in genes with undefined cancer risks or P/LP variants in genes that lack established screening guidelines. ${ }^{23}$ Another concern is the risk of overestimating the clinical implications of a P/LP result in a low- to moderate-risk gene. ${ }^{24} \mathrm{~A}$ further criticism is the proportionate increase in the number of VUS for which management is unclear. Finally, multi-gene panels can require more extensive pre-test genetic counseling. ${ }^{22}$

Despite these limitations, the decreasing cost of genetic testing due to NGS technology has made multi-gene panels accessible to clinicians and patients. In this study, the price of ordering a genetic test was the same regardless of the number of genes on the panel, making test selection dependent only on patient and provider preferences. The majority $(85.6 \%)$ of our cohort of 1085 patients were tested with broader panels that assessed risks for multiple cancer types (groups B and C). This result is consistent with the reported trend of providers ordering testing of more genes per patient. $^{25}$

This study had a number of limitations. Although published management guidelines exist for the majority of positive variants identified, the utility of discovering these variants for post-test management was assumed but not assessed. Further analysis of patient outcomes is needed to clarify the actual clinical impact of panel testing. Because panel selection was at the discretion of the ordering provider, the genes analyzed varied by patient. This may have been influenced in part by the tendency of clinicians to order a large panel for patients with complicated clinical presentations. However, this was not assessed in our study. In addition, personal and family history information was limited to that on the test request form. Any relevant family history or secondary diagnosis was not analyzed. Finally, we were unable to assess ordering patterns by clinician type (genetic counselor, surgeons, oncologists, OB/gyn).

\section{CONCLUSION}

Expanded panel testing increased the identification of P/LP findings related to non-breast cancers. Notably, most of these findings occurred in clinically actionable genes with published management recommendations.

The potential downsides of comprehensive panel testing must be weighed against the opportunity to discover actionable variants that may lead to earlier screening and detection, prevention, and decreased cancer morbidity. Pretest counseling should address the potential for incidental but actionable findings and findings that have no medical management guidelines. Finally, further analysis is needed to determine the clinical impact and patient outcomes associated with the identification of P/LP variants in non-BRCAl/2 genes.

ACKNOWLEDGMENT We thank Robert Nussbaum, MD, Nancy Jacoby, and Kristen McCaleb, PhD for their contributions and review of the manuscript. 
DISCLOSURE All the authors are affiliated with Invitae Corp.

OPEN ACCESS This article is distributed under the terms of the Creative Commons Attribution 4.0 International License (http:// creativecommons.org/licenses/by/4.0/), which permits unrestricted use, distribution, and reproduction in any medium, provided you give appropriate credit to the original author(s) and the source, provide a link to the Creative Commons license, and indicate if changes were made.

\section{REFERENCES}

1. Lichtenstein P, Holm NV, Verkasalo PK, Iliadou A, Kaprio J, Koskenvuo M, et al. Environmental and heritable factors in the causation of cancer: analyses of cohorts of twins from Sweden, Denmark, and Finland. N Engl J Med. 2000;343:78-85.

2. Pharoah PD, Antoniou A, Bobrow M, Zimmern RL, Easton DF, Ponder BA. Polygenic susceptibility to breast cancer and implications for prevention. Nat Genet. 2002;31:33-6.

3. NCCN Clinical Practice Guidelines in Oncology (NCCN Guidelines). Genetic/familial high-risk assessment: breast and ovarian. Version 2.2017. https://www.nccn.org/. Accessed 31 March 2017.

4. NCCN Clinical Practice Guidelines in Oncology (NCCN Guidelines). Genetic/familial high-risk assessment: colorectal. Version 2.2016. https://www.nccn.org/. Accessed 31 March 2017.

5. The American Society of Breast Surgeons Consensus Guideline on Hereditary Genetic Testing for Patients With and Without Breast Cancer (revised March 14, 2017). https://www. breastsurgeons.org/new_layout/about/statements/PDF_Statements/ BRCA_Testing.pdf. Accessed 1 April 2017.

6. Society of Gynecologic Oncology Clinical Practice Statement: Genetic Testing for Ovarian Cancer. Retrieved 31 March 2017 at https://www.sgo.org/clinical-practice/guidelines/genetic-testingfor-ovarian-cancer/.

7. American College of Obstetrics and Gynecologists, et al. ACOG Practice Bulletin no. 103: Hereditary breast and ovarian cancer syndrome. Obstet Gynecol. 2009;113:957-66.

8. Maxwell KN, Domchek SM. Cancer treatment according to BRCA1 and BRCA2 mutations. Nat Rev Clin Oncol. 2012;9: 520-8.

9. Tinat J, Bougeard G, Baert-Desurmont S, Vasseur S, Martin C, Bouvignies E, et al. 2009 version of the Chompret criteria for $\mathrm{Li}$ Fraumeni syndrome. J Clin Oncol. 2009;27:e108-9.

10. Pilarski R, Burt R, Kohlman W, Pho L, Shannon KM, Swisher E. Cowden syndrome and the PTEN hamartoma tumor syndrome: systematic review and revised diagnostic criteria. J Natl Cancer Inst. 2013;105:1607-16.

11. Rehm HL. Disease-targeted sequencing: a cornerstone in the clinic. Nat Rev Genet. 2013;14:295-300.

12. Tucker T, Marra M, Friedman JM. Massively parallel sequencing: the next big thing in genetic medicine. Am J Hum Genet. 2009;85:142-54.

13. Johansen Taber KA, Dickinson BD, Wilson M. The promise and challenges of next-generation genome sequencing for clinical care. JAMA Intern Med. 2014;174:275-80.

14. Kurian AW, Hare EE, Mills MA, Kingham KE, McPherson L, Whittemore AS, et al. Clinical evaluation of a multiple gene sequencing panel for hereditary cancer risk assessment. $J$ Clin Oncol. 2014;32:2001-9.

15. Tung N, Battelli C, Allen B, Kaldate R, Bhatnagar S, Bowles K, et al. Frequency of mutations in individuals with breast cancer referred for $B R C A 1$ and $B R C A 2$ testing using next-generation sequencing with a 25-gene panel. Cancer. 2015;121:25-33.

16. LaDuca H, Stuenkel AJ, Dolinsky JS, Keiles S, Tandy S, Pesaran $\mathrm{T}$, et al. Utilization of multigene panels in hereditary cancer predisposition testing: analysis of more than 2000 patients. Genet Med. 2014;16:830-7.

17. Maxwell KN, Wubbenhorst B, D'Andrea K, Garman B, Long $\mathrm{JM}$, Powers J, et al. Prevalence of mutations in a panel of breast cancer susceptibility genes in BRCA1/2-negative patients with early-onset breast cancer. Genet Med. 2015;17:630-8.

18. Lincoln SE, Kobayashi Y, Anderson MJ, Yang S, Desmond AJ, Mills MA, et al. A systematic comparison of traditional and multigene panel testing for hereditary breast and ovarian cancer genes in more than 1000 patients. J Mol Diagn. 2015;17:533-44.

19. Nykamp K, Anderson M, Powers M, Garcia J, Herrera B, Ho, Y, et al. Sherloc: a comprehensive refinement of the ACMG-AMP variant classification criteria. Genet Med. DOI:10.1038/gim.2017. 37.

20. Hall MJ, Forman AD, Pilarski R, Wiesner G, Giri VN. Gene panel testing for inherited cancer risk. J Natl Compr Cancer Netw. 2014;12(9):1339-46.

21. Mauer CB, Pirzadeh-Miller SM, Robinson LD, Euhus DM. The integration of next-generation sequencing panels in the clinical cancer genetics practice: an institutional experience. Genet Med. 2014; $16: 407-12$.

22. Fecteau H, Vogel KJ, Hanson K, Morrill-Cornelius S. The evolution of cancer risk assessment in the era of next generation sequencing. J Genet Couns. 2014;23:633-9.

23. Easton DF, Pharoah PD, Antoniou AC, Tischkowitz M, Tavtigian SV, Nathanson KL, et al. Gene-panel sequencing and the prediction of breast-cancer risk. $N$ Engl J Med. 2015;372:2243-57.

24. Thompson ER, Rowley SM, Li N, McInerny S, Devereux L, Wong-Brown MW, et al. Panel testing for familial breast cancer: calibrating the tension between research and clinical care. Rev Assoc Med Bras. 2015:61:282-9.

25. Blazer KR, Nehoray B, Solomon I, Niell-Swiller M, Culver JO, Uman GC, Weitzel JN. Next-generation testing for cancer risk: perceptions, experiences, and needs among early adopters in community healthcare settings. Genet Test Mol Biomarkers. 2015;19:657-65.

26. Slavin TP, Niell-Swiller M, Solomon I, Nehoray B, Rybak C, Blazer KR, Weitzel JN. Clinical application of multigene panels: challenges of next-generation counseling and cancer risk management. Front Oncol. 2015;5:208.

27. Howarth DR, Lum SS, Esquivel P, Garberoglio CA, Senthil M, Solomon NL. Initial results of multigene panel testing for hereditary breast and ovarian cancer and Lynch syndrome. $\mathrm{Am}$ Surg. 2015;81:941-4.

28. Desmond A, Kurian AW, Gabree M, Mills MA, Anderson MJ, Kobayashi Y, et al. Clinical actionability of multigene panel testing for hereditary breast and ovarian cancer risk assessment. JAMA Oncol. 2015;1:943-51.

29. Castéra L, Krieger S, Rousselin A, Legros A, Baumann JJ, Bruet $\mathrm{O}$, et al. Next-generation sequencing for the diagnosis of hereditary breast and ovarian cancer using genomic capture targeting multiple candidate genes. Eur J Hum Genet. 2014;22:1305-13.

30. Thompson ER, Rowley SM, Li N, McInerny S, Devereux L, Wong-Brown MW, et al. Panel testing for familial breast cancer: calibrating the tension between research and clinical care. J Clin Oncol. 2016;34:1455-9.

31. Maxwell KN, Hart SN, Vijai J, Schrader KA, Slavin TP, Thomas $\mathrm{T}$, et al. Evaluation of ACMG-guideline-based variant classification of cancer susceptibility and non-cancer-associated genes in families affected by breast cancer. Am J Hum Genet. 2016;98:801-17. 
32. Couch FJ, Hart SN, Sharma P, Toland AE, Wang X, Miron P, et al. Inherited mutations in 17 breast cancer susceptibility genes among a large triple-negative breast cancer cohort unselected for family history of breast cancer. J Clin Oncol. 2015;33:304-11.

33. Cleary SP, Cotterchio M, Jenkins MA, Kim H, Bristow R, Green $\mathrm{R}$, et al. Germline MutY human homologue mutations and colorectal cancer: a multisite case-control study. Gastroenterology. 2009;136:1251-60.

34. Jones S, Emmerson P, Maynard J, Best JM, Jordan S, Williams GT, et al. Biallelic germline mutations in MYH predispose to multiple colorectal adenoma and somatic $\mathrm{G}: \mathrm{C}->\mathrm{T}: \mathrm{A}$ mutations. Hum Mol Genet. 2002;11:2961-7.

35. Al-Tassan N, Chmiel NH, Maynard J, Fleming N, Livingston AL, Williams GT, et al. Inherited variants of MYH associated with somatic G:C->T:A mutations in colorectal tumors. Nat Genet. 2002;30:227-32.

36. Hale V, Weischer M, Park JY. CHEK2 (*) 1100delC mutation and risk of prostate cancer. Prostate Cancer. 2014;2014:294575.
37. Cybulski C, Wokołorczyk D, Jakubowska A, Huzarski T, Byrski $\mathrm{T}$, Gronwald J, et al. Risk of breast cancer in women with a CHEK2 mutation with and without a family history of breast cancer. J Clin Oncol. 2011;29:3747-52.

38. Xiang HP, Geng XP, Ge WW, Li H. Meta-analysis of CHEK2 1100 delC variant and colorectal cancer susceptibility. Eur J Cancer. 2011;47:2546-51.

39. Wasielewski M, den Bakker MA, van den Ouweland A, Meijervan Gelder ME, Portengen H, Klijn JG, et al. CHEK2 1100delC and male breast cancer in the Netherlands. Breast Cancer Res Treat. 2009;116:397-400.

40. Cybulski C, Górski B, Huzarski T, Masojć B, Mierzejewski M, Debniak T, et al. CHEK2 is a multiorgan cancer susceptibility gene. Am J Hum Genet. 2004;75:1131-5.

41. Narod SA. Testing for CHEK2 in the cancer genetics clinic: ready for prime time? Clin Genet. 2010;78:1-7.

42. Mahon SM. Breast cancer risk associated with CHEK2 mutations. Oncol Nurs Forum. 2014;41:692-4. 